\title{
Three Dimensional Tangible User Interface for Controlling a Robotic Team
}

\author{
Paul Lapides \\ University of Calgary \\ 2500 University Drive NW, \\ Calgary, Alberta, Canada \\ paul.lapides@gmail.com
}

\author{
Ehud Sharlin \\ University of Calgary \\ 2500 University Drive NW, \\ Calgary, Alberta, Canada \\ $+1.403 .210 .9404$ \\ ehud@cpsc.ucalgary.ca
}

\author{
Mario Costa Sousa \\ University of Calgary \\ 2500 University Drive NW, \\ Calgary, Alberta, Canada \\ $+1.403 .220 .6783$ \\ mario@cpsc.ucalgary.ca
}

\begin{abstract}
We describe a new method for controlling a group of robots in three-dimensional (3D) space using a tangible user interface called the 3D Tractus. Our interface maps the task space into an interactive $3 \mathrm{D}$ space, allowing a single user to intuitively monitor and control a group of robots. We present the use of the interface in controlling a group of virtual software bots and a physical Sony AIBO robot dog in a simulated Explosive Ordnance Disposal (EOD) environment involving a bomb hidden inside of a building. We also describe a comparative user study we performed where participants were asked to use both the 3D physical interface and a traditional 2D graphical user interface in order to try and demonstrate the benefits and drawbacks of each approach for HRI tasks.
\end{abstract}

\section{Categories and Subject Descriptors}

H.5.2 [Information interfaces and presentation]: user interfaces - interaction styles, graphical user interfaces (GUI), theory and methods

\section{General Terms}

Design, Experimentation, Human factors

\section{Keywords}

Human-robot interaction, tangible user interfaces, physical interfaces, interaction techniques, evaluation, robotic team control, Explosive Ordnance Disposal (EOD)

\section{INTRODUCTION}

Efficient interfaces for controlling robots are still an open humanrobot interaction (HRI) research problem. The challenge of controlling robots scales dramatically when considering tasks of shared interaction [16] between a single human operator and a team of multiple independent robots. Another more general challenge in human-computer interaction (HCI) is providing three-dimensional (3D) awareness of spatial interactive tasks. In

Permission to make digital or hard copies of all or part of this work for personal or classroom use is granted without fee provided that copies are not made or distributed for profit or commercial advantage and that copies bear this notice and the full citation on the first page. To copy otherwise, or republish, to post on servers or to redistribute to lists, requires prior specific permission and/or a fee.

HRI'08, March 12-15, 2008, Amsterdam, Netherlands.

Copyright 2008 ACM 978-1-60558-017-3/08/03..\$5.00. this paper we address both challenges through the design, implementation and comparative evaluation of a new interface for controlling a group of robots in 3D space.

We designed a physical interface called the 3D Tractus that enables control of a group of robots in 3D space using a physical drawing board interaction metaphor. The 3D Tractus was designed with 3D spatial tangible user interfaces (TUIs) themes in mind. The interface allows direct mapping between the interaction space to a 3D physical task space, such as air space in the case of unmanned aerial vehicles (UAVs), or buildings in the case of urban search and rescue (USAR) or Explosive Ordnance Disposal (EOD) robotic tasks. The user can interact in the 3D domain by physically sliding the 3D Tractus surface up and down in space. The 3D Tractus enables control of the robotic team on the interactive surface via simple sketch-based stylus actions. Overall, the 3D Tractus provides the user with intuitive spatial visualization of the task state as well as with tools for intuitively monitoring and distributing tasks between the robotic team members.

In this paper we describe the 3D Tractus-based robotic interface, with its current use for controlling a group of robots composed of independent AIBO robot dogs and virtual software entities. We describe our experimental test bed, a game-like 3D EOD environment in which a group of robots are controlled by a single human operator in a searching task (Figure 1). The user is asked to control the robotic team and explore a building in an attempt to find and defuse a hidden bomb as quickly as possible. Our current test bed is highly idealized. We assume perfect a priori knowledge of the environment and the robots location, in order to allow us to
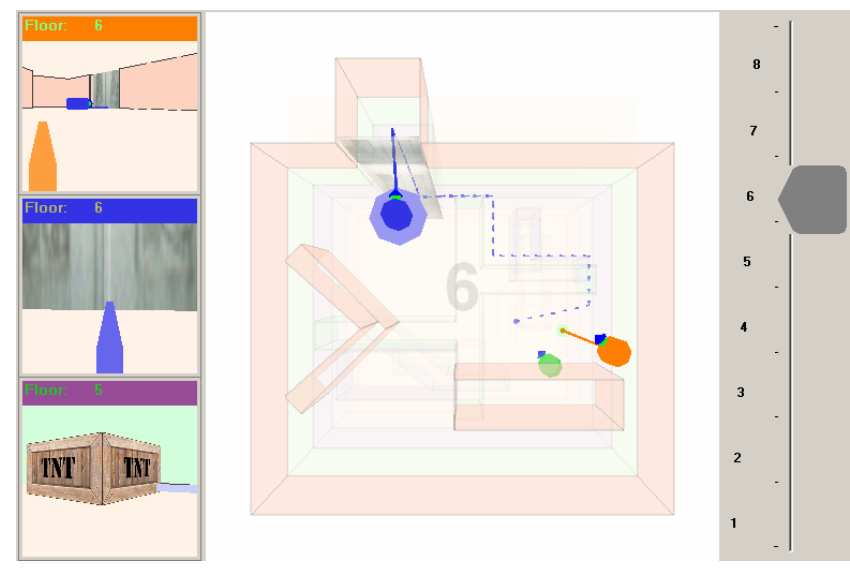

Figure 1: Robots can move in 3D through a building. 
pursue a set of elementary questions: is our TUI useful for robotic group control in 3D space? Would the physical 3D Tractus be more efficient for the team control task compared to a similar non-physical graphical user interface (GUI)? While the validity of our current test bed EOD task is arguably low, we believe that the near future will provide strong motivations for efficient 3D spatial robotic group control. Examples may range from mining tasks, space exploration, UAVs or Unmanned Undersea Vehicles (UUV). If our thesis is correct, physical TUIs such as the 3D Tractus can help reduce the ratio of users per robots in such tasks, and offer intuitive mapping between the robotic group 3D task space and the user's interaction space.

In order to explore our thesis we conducted a comparative study, mapping two interfaces to our experimental test bed environment: the first is the Tractus TUI and the second is a Window-IconMenu-Pointer (WIMP) slider. This paper discusses our experimental design, the pilot study phase and a full study we performed comparing the two interfaces.

Our contribution in this paper is twofold: the design of a new physical TUI for 3D spatial control of a group of robots by a single user; and a user study comparing the interaction quality of the physical TUI to a non-physical GUI-based parallel interface in a simulated EOD-like environment.

\section{RELATED WORK}

Our work relates to the fundamental challenge of controlling a team of independent robots by a single user. Following Yanco and Drury's taxonomy [16] our implementation can be useful for tasks with a high level of user intervention and with one human controlling a group of multiple independent robots.

Several previous PDA-based sketch-based interface systems for robot control have been proposed and can be categorized as relying on either quantitative $[2,7,8]$ or qualitative $[1,3,10] 2 \mathrm{D}$ maps.

Recently, Skubic et al [12] proposed a sketch-based interface to control multiple robots using a qualitative, hand-drawn map. The paper proposes a single user, Tablet PC sketch-based interface for controlling a team of robots along a sketched 2D route map. In their system, the user sketches a qualitative map of the environment describing the scene, and then uses navigation gestures to direct the robots. The interface also displays the robot's sensor feedback and allows the synchronization of robot locations. A usability study was conducted in two groups, one controlling the robots in an unaltered environment and the other group in a slightly altered environment. Most users positively validated the sketch-based interface for guiding mobile robots.

We designed the 3D Tractus as a TUI. TUIs are interfaces that couple digital information and function with physical artifacts [15]. Good spatial TUIs will provide intuitive spatial mappings between the physical object and the task, unification of input and output spaces and support of trial and error activity [11].

The 3D Tractus is a simple TUI that was originally designed for general 3D sketch-based interaction [5] and for plant modeling [13]. The user can move the 3D Tractus vertically up and down while sketching on a tablet PC placed on its surface. The 3D Tractus height is being tracked using a simple sensor and the stylus surface position is tracked through a tablet PC or any other touch sensitive surface interface [5]. The 3D Tractus was designed to support direct mapping between its physical space to the task virtual space, and can be viewed as a minimal and inexpensive sketch-based variant of the Boom Chameleon [14].

As far as we know, our work is one of the first to explore the use of TUIs in HRI applications. In a pioneering effort, Quigley, Goodrich and Beard explored [9] the use of a physical icon (phicon [15]) for intuitive control of a mini-UAV, empirically comparing the phicon controller to a number of other, non-TUI control mechanisms. Guo and Sharlin [4] mapped a generic Wiimotes-based TUI to an AIBO robot dog in various locomotion and posture control tasks, comparing the TUI effectiveness to a keyboard controller. The work presented in this paper is unique in its use of a 3D sketch based spatial TUI for human-robot interaction tasks, supporting robotic group control mechanisms, and empirically comparing the TUI effectiveness to a non-TUI 2D sketch-based controller.

\section{SYSTEM DESCRIPTION 3.1 Overview}

Our application enables controlling a group of robots in 3D from a high-level perspective. The user takes the role of team leader and gives macro instructions to the group, overseeing the overall progress of the robots. The system intentionally avoids micro details like exact locomotion guidance in order to focus the users' attention on the group management and to allow explicit investigation of our TUI vs. GUI research question.

We chose to orient the application as a game to demonstrate the power of our system and to make it fun to use during the user studies. In the game, a group of robots are dispatched into a building and are hunting for a bomb hidden inside. Robots are fitted with cameras that show their surroundings on the screen. The user must pay attention to these video feeds and once the bomb has been sighted they must guide the robots to the bomb in order to defuse it (Figure 1).

In the first iteration of the system, the entire scenario was simulated on the computer like a traditional video game. The building is an arbitrary arrangement of floors stored as a file with special locations for an elevator and bomb. The robots are also virtual entities and obey basic physical laws as real robots would. For example, they cannot move through walls and have realistic speeds for locomotion. Robots can only perform simple actions like turning and moving forward.

In the second iteration of the system, we integrated a Sony AIBO ERS-7M2 robotic dog into the scenario to show that our interface can be used to control real robots and not just simulated entities. The AIBO satisfied the minimum requirements for our environment because it can turn, move forward, and has a video camera that is used to show its surroundings. The AIBO robot is only a working concept as we currently have not fully implemented tracking. Unlike the virtual entities, the system is not aware of the AIBO's exact position and orientation, meaning that it is behaving as an open-loop system. The AIBO will go off track after a period of time and must be "recalibrated" by aligning the real robot with its virtual representation.

The user gives instructions to the robots by specifying a place in the building they should move to during the EOD task. The building has several floors so the user must be able to move up and down through the building to access all the floors. We 
implemented two interfaces that give the user the 3D interaction abilities needed to move vertically throughout the building. The first interface is a traditional WIMP slider where the vertical position of the slider determines what floor of the building the user is currently viewing. The second interface is a TUI we developed called the 3D Tractus [5]. It is a movable table surface with a tablet PC running the application lying on top. The user slides the table up and down physically changing their viewpoint inside the building and clicking on the surface to issue robot tasks.

The application can run on any Windows PC and requires only a mouse (or stylus) with left-click functionality. We have combined the output (screen) and input (mouse cursor) context spaces into a single space by running the application on a Toshiba Portege M200 tablet PC and using the stylus to click the screen. The application is written in $\mathrm{C \#}$ using the CsGL OpenGL wrapper library.

\subsection{Controlling Robots}

The game has three important areas on the screen shown in Figure 1. In the middle is a top-down view of the building showing the floor the user is currently viewing. This is where the robots' locations are displayed and instructions are given by clicking on the floor. On the left are the video feeds from each robot in the building. Each video feed has a color bar corresponding to the robot the video is coming from, as well as a number showing what floor the robot is on. The user must watch these feeds in order to identify the bomb when a robot passes near it. Finally, on the right is the vertical slider that controls the user's position in the building. In the slider mode the user controls the slider by directly dragging it up and down, and in the Tractus mode the slider is controlled by the physical height of the Tractus (when the Tractus is moved up, the slider will move up, etc.)

Robots are selected and deselected by clicking on them once. Once a robot is selected, a place on the floor is clicked and the robot automatically moves to that location; this is called a task.

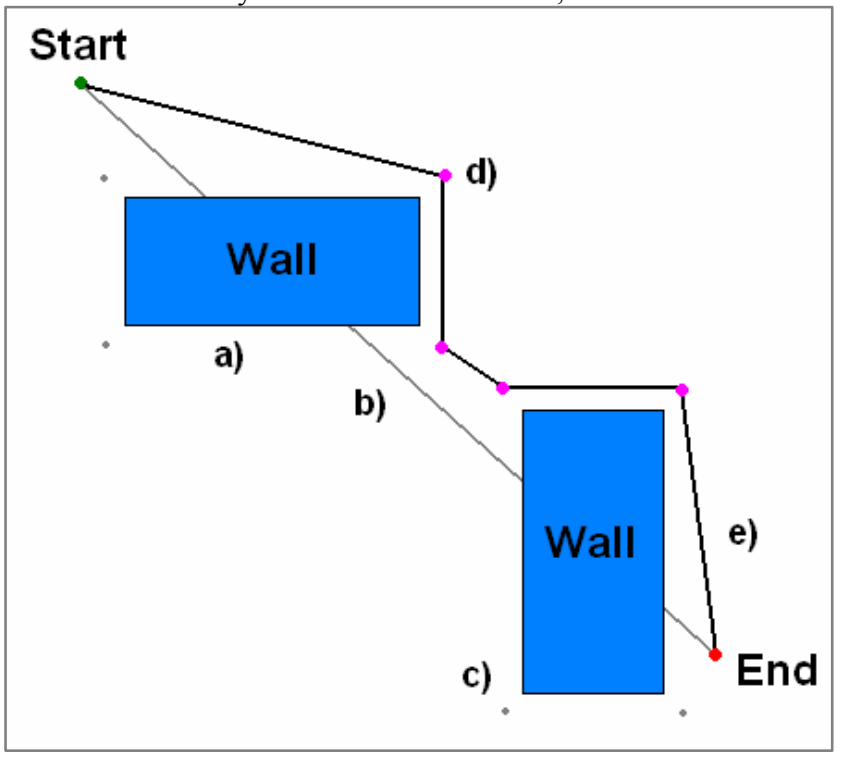

Figure 2: Guidance algorithm. a) Obstacles, b) Proposed path (occluded by walls), c) Margin points for walls, d) Intermediate tasks (margin points), e) New path created for the robot to follow.
The user can keep queuing tasks after the first task is issued and the robot will move to the locations in the order they were given. The user can clear the robot's queued tasks by reselecting the robot (clicking on the robot again). By selecting more than one robot before issuing tasks, multiple robots will move in unison.

To allow our EOD robots to move vertically between floors we chose a simplified solution in the form of an elevator. To move between floors, the robot is selected as before and the user changes floors using either the slider or the Tractus. Once the desired floor is reached, the user clicks somewhere on the floor and a task is issued. The robot will automatically find the elevator, move vertically to the target floor, and then move to the designated destination.

The robots must find a single bomb that is represented by a wooden crate labeled "TNT". This object easily stands out from the building and is easy to spot on the robots' video feeds. A robot does not change its behavior when the bomb is in sight but continues following the tasks that were assigned. It is the user's responsibility to navigate the robot to the bomb once they discover where it is hidden. If a robot is within a small distance of the bomb ( 1 meter in comparative terms) it will automatically stop and begin defusing the bomb. The color bar above the robot's video feed will begin blinking to notify the user of the robot's success and the robot will not respond to any more commands. Once the bomb is located, the user must guide all the robots to the bomb. Only when all the robots dispatched in the building are engaged in defusing does the bomb become fully disarmed.

\subsection{Automatic Guidance}

Our system is designed to let the user manage the robotic team from a high-level role and free them from micromanaging each robot. In order to accomplish this, the robots must be intelligent enough to navigate through moderately complex environments to reach their issued destinations. Floors are rarely just rectangular rooms but are full of objects like chairs, desks, and other walls. For simplicity, the only kind of obstacle in the game is a wall, though other objects can easily be added. The robots have a basic AI path finding algorithm to guarantee that they can reach any point on an arbitrary floor.

The guidance system is used when a given task does not have an unobstructed line from the start to the end; in other words, there is a wall in the way of the robot. The algorithm, schematically shown in Figure 2, creates intermediary tasks around the corners of the wall. The algorithm is recursive, so it is able to handle an unlimited number of obstacles. Due to complexity, the algorithm is not optimized to give the shortest possible route. The floor plan of the building is known a priori and the algorithm uses data directly from the layout of the walls.

\subsection{Integrating Real Robots}

The robots described so far are virtual entities handled entirely by the computer. The virtual robots only have to rotate, move forward, and provide a video feed, all of which is handled by the software. As long as these three requirements are satisfied, physical robots can be integrated into the game and be controlled with the same interaction metaphors as before.

To demonstrate this feature, we programmed an AIBO robot to be controlled wirelessly by the game. The software sends rotation and walking commands to the dog and in return the AIBO sends back JPEG frames from its built in webcam. There is currently no 


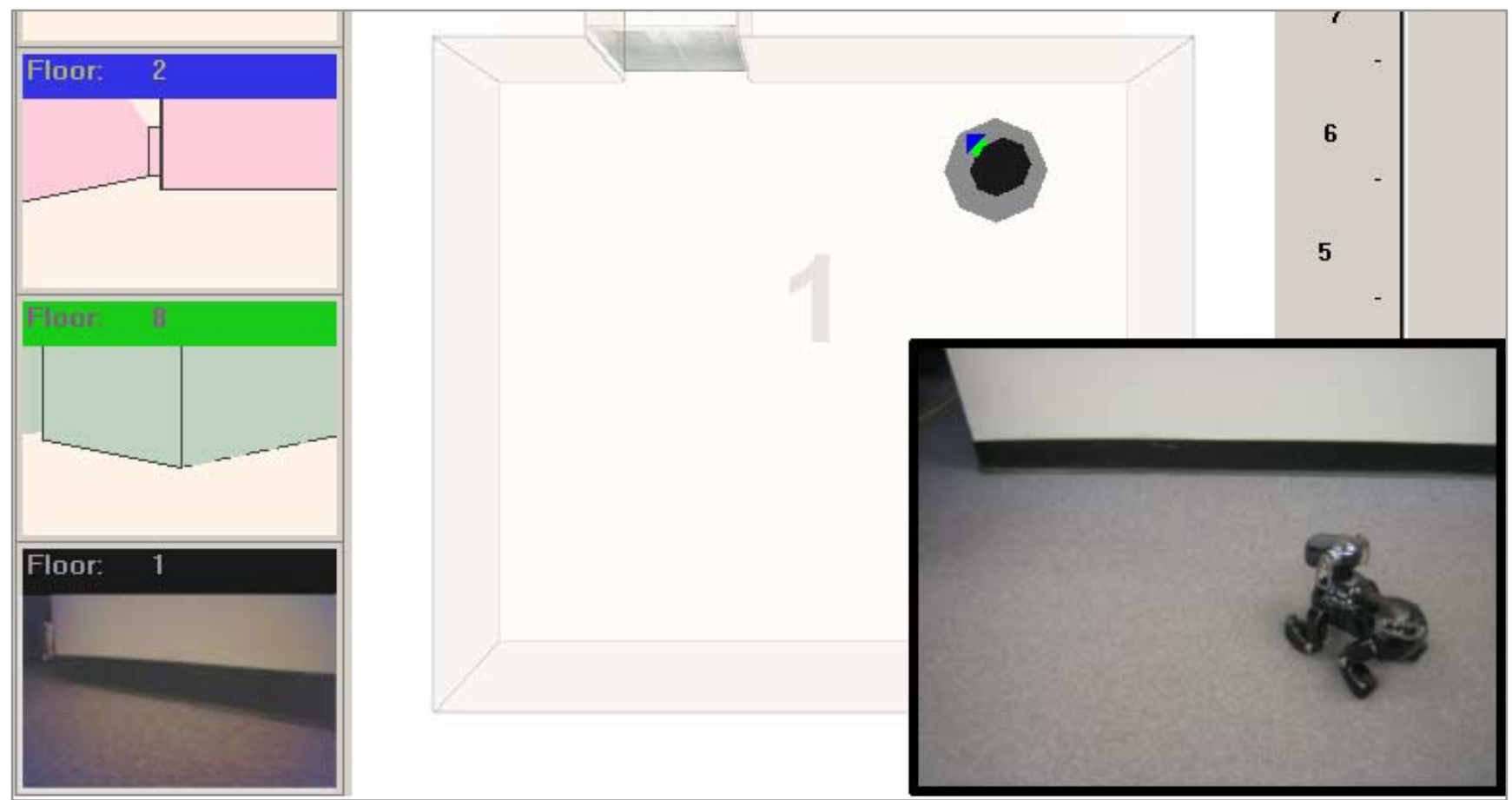

Figure 3: An AIBO dog is integrated into the system and has a real video feed, shown on the bottom left.

positional feedback that updates the AIBO's virtual avatar in the game. In other words, this is an open-loop system and the dog is not being tracked. As far as the game is concerned, the AIBO entity is just another virtual robot and the game is in control of moving and guiding it on the screen (Figure 3 ).

The turning and moving speeds of the virtual entity representing the AIBO have been calibrated to approximately the same speeds as the AIBO's physical movements. This means that the positions of the AIBO and its virtual avatar are consistent for the first few minutes of the game, but eventually deteriorate because the AIBO's actual movements are not being tracked.

\subsection{Visualization}

Several visualization cues are used in the top-down view of the game. The floors of the building are transparent allowing the user to see the floors below. This is done to show more information about the locations and tasks of the robots below the current floor. Using the Tractus, this merges the virtual and physical spaces by visually conveying the notion that there is actually something below the current level of the 3D Tractus's surface. The building view is top-down so it would not be logical to show the floors above the current height.

Making the floors transparent carries the risk of overloading the user with too much information and cluttering the interface. The transparency is controlled by a non-linear gradient, so floors just below the current height are still visible while floors far away are completely hidden (Figure 4). The displayed size of the robot entities follows the same gradient: the further away from the current height a robot gets, the smaller it is drawn so the user is less likely to be confused regarding which robots are on the current floor. When selected, robots are highlighted and start to pulsate to clearly distinguish them from unselected entities.
The task lines (Figure 4) have the highest potential to clutter the screen. If there are many lines, they may block objects behind them and can potentially confuse the user about which robot is going where. It must also be clear in what direction the task line is pointing and how far it is ordered in the robot's execution queue. The first visualization that is applied to the task lines is the same as the floor transparency. The further lines get away from the current height the less opaque they become, until they eventually disappear at a certain distance. This prevents the view from being cluttered by task lines that are far below the current height. The second visualization, shown in Figure 5, is that lines are dashed

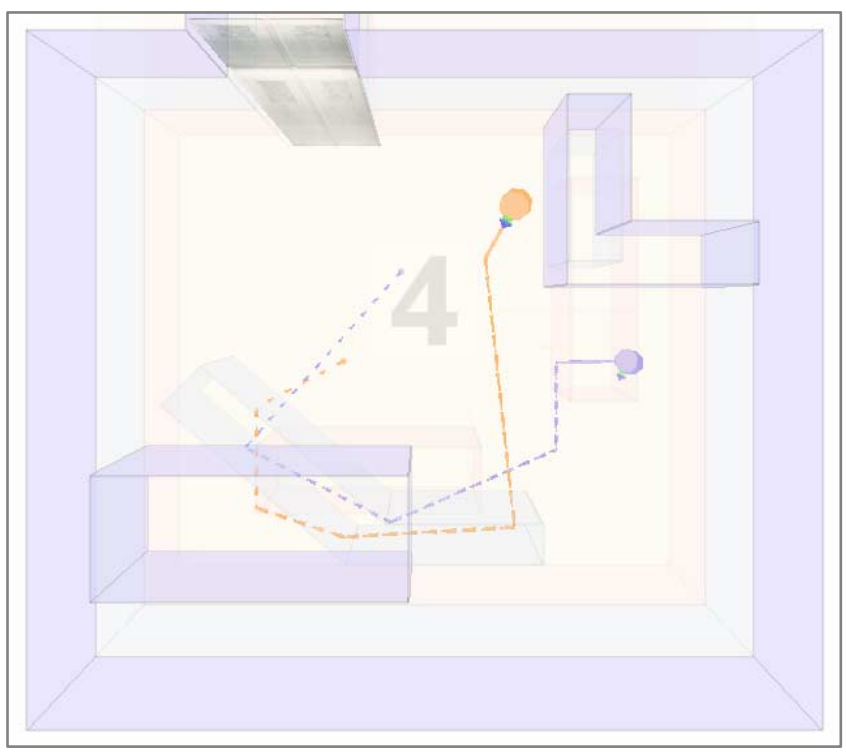

Figure 4: Transparent floors and tasks. 


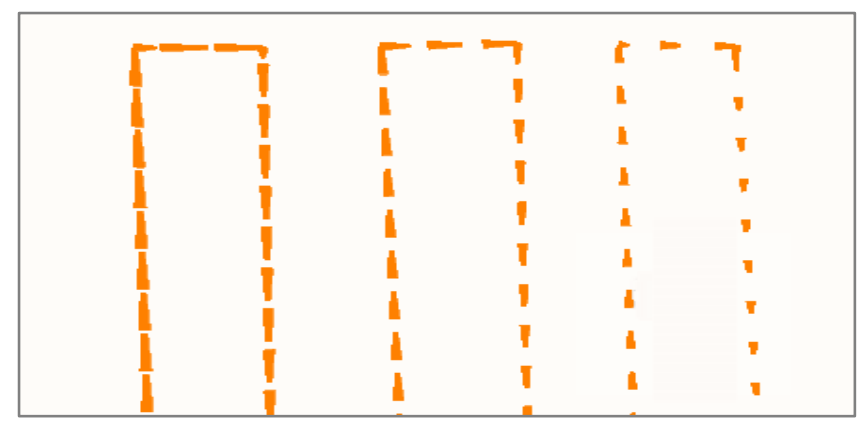

Figure 5: Task queue; task lines are sparsely dashed to show when thev will be executed.

more sparsely when they are further in the execution queue, to show that the task is not about to be executed soon. The dashes are arrow shaped to show the direction of the task.

\section{INTERFACES}

Two different interfaces are used to the control the vertical movement in the game, enabling robots to move in 3D. The first is a traditional WIMP GUI slider and the second is a tangible device called the 3D Tractus.

\subsection{Slider}

A vertical slider is shown on the right side of the screen. In this interaction mode, the user drags the slider up and down with the mouse to control the height in the building. This slider differs slightly from the standard Windows slider because the sliding widget is significantly enlarged, making it easier to click when the user wants to slide quickly.

\subsection{D Tractus}

The 3D Tractus is a simple mechanical device consisting of a table surface that slides up and down on four vertical tracks [5]. The frame, shown in Figure 6, is constructed from $80 / 20$ Industrial Erector Set aluminum bars and can be easily assembled with a screwdriver. The application runs on a Toshiba Portege M200 tablet PC that lies on top of the table surface. The user moves the table surface up and down using their spare hand while either sitting or standing. A counterweight system similar to those used in elevators keeps the table surface balanced so the user does not have to support the weight of the tablet with their arm and makes it easier to move the surface up and down. The maximum vertical range of the surface is about $35 \mathrm{~cm}$ but only an $18 \mathrm{~cm}$ range is needed for operation in this case.

A string potentiometer measures the height of the table by increasing the voltage on its output when the string is extended and decreasing the voltage when retracted. An Ontrak ADU100 Data Acquisition Device (analog/digital converter) converts the voltage to a 16-bit unsigned integer. The ADU100 communicates with Windows via USB and the height of the table surface is sampled in real time.

Because the user is looking down on the screen of the tablet when using the 3D Tractus, the top-down view of the building in the game combines the physical and virtual spaces providing a visual illusion that the building exists in the physical world.

\section{USER STUDY}

To gain insight into our TUI approach to robotic group interaction we designed a comparative experimental test bed that asks the user to find a hidden bomb in the building during several trials, alternating between the TUI and the slider conditions. Our goal was to reveal the benefits and drawbacks of each interaction method and to learn which method supports intuitive and effective control of a group of robots.

\subsection{Experimental Design}

Each trail in the study began with an explanation of the purpose of the study and an introduction to the interface. Participants then received training on how to move vertically in the building using either the slider or the Tractus, how to control the robots, and how to defuse the bomb. They were then asked to defuse a bomb in a short training exercise involving two robots called Orange and Navy (corresponding to their colors) in a two-storey building with various obstacles. The bomb was hidden behind a wall on the $2^{\text {nd }}$ floor. Once the user was comfortable with the game, the actual experiment was revealed to them.

The user performed three trials with the bomb hidden in a different place each time. Users controlled three robots (Orange, Navy, and Forest) in an eight-storey building. The Orange and Navy robots started on the first floor and Forest started on the eighth floor. The user guided the robots inside the building until the bomb was located and defused by moving all three robots close to the bomb, at which point the trial was complete. The bomb was hidden on the $4^{\text {th }}$ floor for trial 1 and in different places on the $5^{\text {th }}$ floor for trials 2 and 3 . For each trial the bomb is hidden
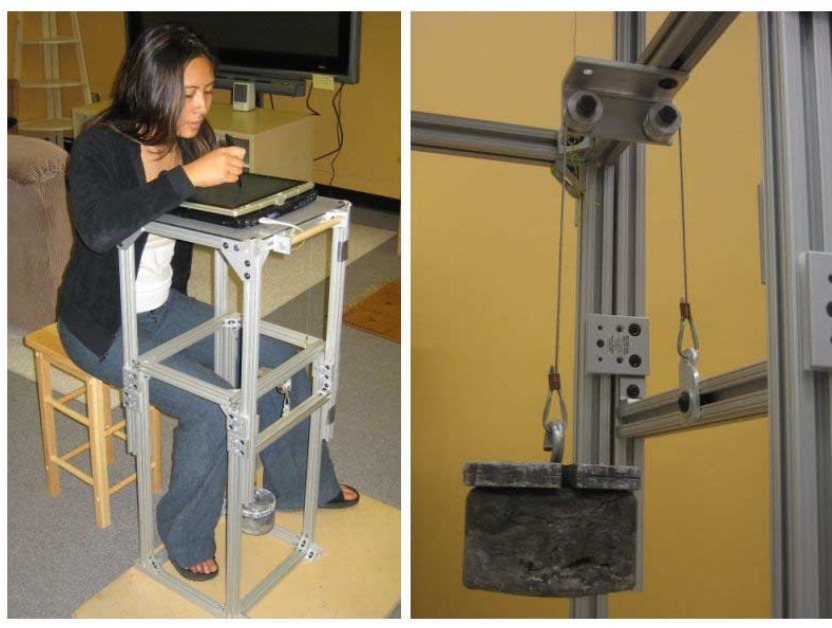

Figure 6: The 3D Tractus frame and counter-weight.

behind a wall, forcing the user to explore each floor to be sure that they have not missed the bomb.

Once the three trials were complete, the user was trained to use the alternative interface. If they first used the slider they would be shown how to use the Tractus, and vice versa. The user was then asked to defuse a bomb in the same two-storey building as in the first training exercise, with the same robots. The bomb was hidden behind a wall in a different place on the $2^{\text {nd }}$ floor. Next the user did three more trials with the same eight-storey building and the same three robots as in the first three trials. The bomb was 
hidden on the $6^{\text {th }}$ floor for trial 1 , the $4^{\text {th }}$ floor for trial 2 , and the $5^{\text {th }}$ floor for trial 3, all behind walls.

After the last three trials were completed, the user filled in a questionnaire that asked them to rate their understanding and performance in the game, as well as pick an overall favorite interaction mode (slider or Tractus).

As participants play the game, they learn the layout of the building, which certainly influences their performance by allowing them to better plan where to send robots and by giving them general practice. We avoided bias to either of the interaction modes by counter-balancing the interface order. Half of the participants first used the slider and half first used the Tractus. The positions of the bombs remained the same regardless of what mode was used first. That is, on the same trial number (1 to 6), a participant using the slider first will find the bomb in the same place as a participant using the Tractus first.

As the user searches for the bomb, the experimental test bed logs all actions that the user performs within the game environment. Everything from selecting robots, to changing floors, to giving tasks is logged in a file with timestamps and other attributes. The files are later analyzed and data is extracted for each participant on a per trial basis. For example, numbers are extracted for the time it takes to defuse the bomb, the number of robot selections made, the number of tasks given, etc.

\subsection{Pilot and Full Study}

A pilot study followed by a full user study were conducted comparing the effectiveness of the slider interaction mode to the Tractus interaction mode. Participants were recruited by sending notices to mailing lists in the Computer Science and Electrical Engineering departments at the University of Calgary.

The pilot study was conducted with 6 participants who were experienced with computers and had some experience with robots. The pilot study was used to find bugs, adjust the experiment time and balance the difficulty level between the different bomb locations, making them equally difficult to find.

Twenty participants were recruited for the full study; their average age was 22.65 years with a standard deviation of 3.11 years. Most participants were students and had extensive computer experience and moderate video game experience. Participants were compensated $\$ 10$ for their time. The running time of the study was from 45 to 60 minutes.

\section{RESULTS}

Most participants managed to find and defuse the bomb in about 5 minutes, regardless of where the bomb was hidden and the interaction condition. Several participants did not notice the bomb when it appeared on the robots' video feeds and subsequently had to re-explore part or all the building. The fastest time a bomb was found was 59 seconds and the longest was 669 seconds (11 minutes). Over all the trials and participants, the bomb was found on average in 221 seconds, with a standard deviation of 88 seconds.

The main comparison measure between the two interfaces is the time it takes for the bomb to be found. The times for the Tractus and Slider were found to have a statistically significant difference based on a paired t-test analysis. When the Tractus is used first,

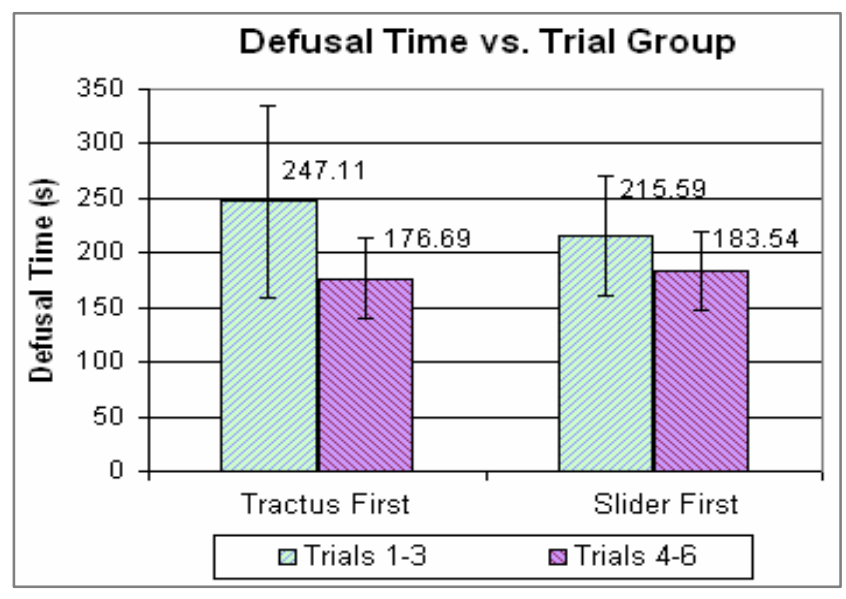

Figure 7: The time until the first robot in the trial finds the bomb and begins defusing against four sets of trials.

the p-value is 0.011 and when the Slider is used first the p-value is 0.041 . The comparison of the mean time to find the bomb is shown in Figure 7.

The number of floor changes made when using the Tractus first and Slider second is statistically different when analyzed using a non-parametric Wilcoxon test, with a p-value of 0.047 . There was no difference when the Slider was used first and Tractus second.

Each participant used each interface with only one set of trials, so it is necessary to determine that the bomb location did not influence the measures. Results for trials 1-3 and 4-6 were found to have no statistically significant difference, and the trial sets were treated identically both for speed and floor changes.

It was found from the questionnaire that exactly half the participants prefer using the slider and half prefer the Tractus. Of the ones that prefer the slider, $50 \%$ actually performed the tasks faster when using the Tractus. 55\% of the participants performed better with the device that they did not prefer, showing no correlation between performance and perceived preference.

\section{DISCUSSION}

It should be reiterated that the purpose of the study was not to test the graphical interface but to compare the slider and Tractus against each other. The interface was purposefully crippled (for example, no audio feedback) in order to encourage people to use the slider and Tractus more. This study gave us an opportunity to look at how people used our system and how we can improve it in the future.

Three types of strategies were observed when the participants controlled the robots. Some users chose to micromanage by giving each robot only one or two tasks at a time and supervising its movement. The user's entire attention was focused on a single robot and this left the other two robots idle most of the time. This type of strategy was inefficient and did not result in very fast search times. On the other extreme, some users tasked the robots to move through every possible area of floor space, relying on the robot's feature of automatically stopping when it gets close to the bomb instead of paying attention to the video feeds themselves. As well, the robots were tasked to many floors at once and the user sat back and watched as the robots executed their instructions. Using this strategy, the user does not play a dynamic 


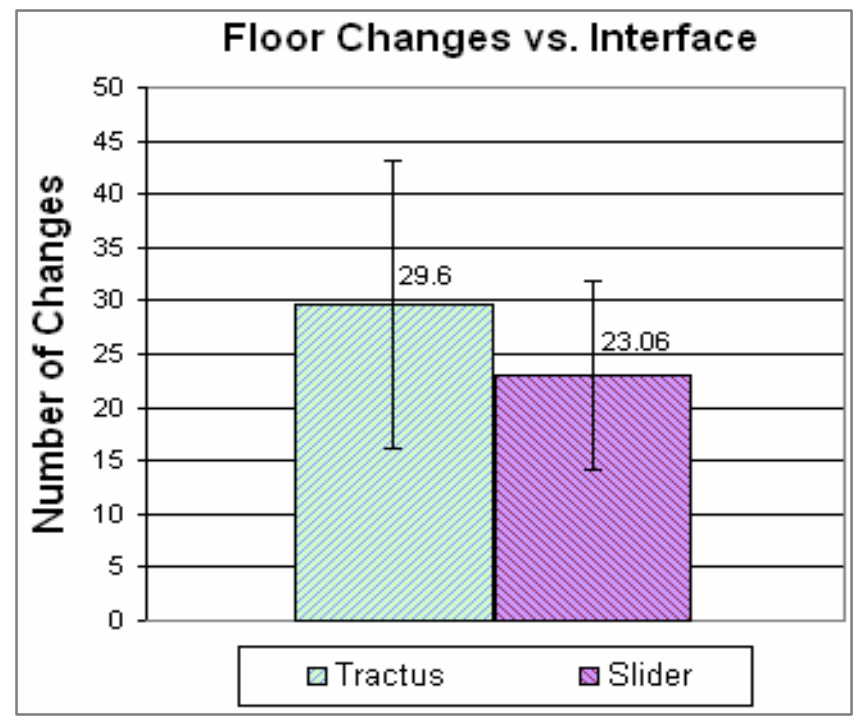

Figure 8: Number of floor changes against trial number for four sets of trials.

role in supervising the robots and does not give the optimum route for the robots by making them scan the entire floor. Users sometimes missed the bomb and had to start searching from the beginning. The largest majority of the users issued many tasks to the robots, usually assigning one robot to a single floor and waiting until the robot was finished searching. The robot was assigned new tasks on an unsearched floor until the bomb was found. This method was the fastest and most efficient because the user watched the video feeds and did not send the robot all over the floor.

Not surprisingly, participants learned to use the interfaces more effectively the more exposure they had to them. Figure 7 shows that the average time to find the bomb is about $20-40 \%$ higher in trials 1-3 than in trials 4-6 regardless of the interface that is first used. As well, the standard deviation of the first three trials is very high. A possible reason for this wide distribution is that participants are unsure of how to use the system most effectively. Three different strategies that participants adopted were just described. Participants could have learned a new strategy that is faster than what they used in the first trials, consequently improving their time, as well as halving the standard deviation during the second set of trials.

The number of floor changes the users makes is a measure that can reflect on how comfortable the user is with the device. Figure 8 shows the number of floor changes with the Tractus and the Slider. If participants change floors too often it can mean that they are unsure of where they are in the building or have trouble moving to the floor they want. However, changing floors less could suggest that users feel restricted by the interface and have to be conservative in their movements. It is difficult to say for certain what the case is with this data, but it is clear that participants have no problem using the Tractus despite its physical nature.

Participants agreed that they were comfortable while using the Tractus based on an average of 4.4 on a 5-point Likert scale [6] (1 is strongly disagree (to being comfortable), 5 is strongly agree). Averages of 4.4 and 4.35 on a 5-point Likert scale ( 1 is strongly disagree, 5 is strongly agree) were found when users where asked if they understand where the robots are in the slider and Tractus trials, respectively, suggesting that both interfaces are equally clear to the user.

A seemingly important reason for participants to prefer the Tractus is because they could use their free hand to change floors instead of having to change context spaces between the robots and the slider. Participants found the Tractus more intuitive and "smoother" than the slider. Several participants suggested a physical slider on the side of the tablet PC be used to change floors instead of the Tractus, which was more "elaborate". A few participants said that keeping track of all the robots and their video feeds was overwhelming and that they were prone to make mistakes and not notice the bomb as easily. Other suggestions were made to have a button deselect all the robots, changing the projection from perspective to orthogonal, showing floors above the current height, and giving the user audio cues when important events occurred (such as the bomb being defused).

Several participants encountered a minor problem when selecting robots. Users would properly select and issue tasks to a robot and then move onto the next robot without deselecting the previous one. The system is designed to operate this way; when the new robot is selected, the old robot is automatically deselected. However, some users were in a rush to select the new robot and "missed" by not clicking on the robot but instead on the floor nearby. The system interprets this as another task being issued and the unwanted task is queued to the old robot. This problem is at best an annoyance because the unwanted task is placed at the very end of the execution queue and does not interfere with the tasks already assigned. The user could tackle this by simply remember that the robot has another task that it will complete, which is something few participants identified. The majority of the users panicked when missing clicks and wasted time clearing and reassigning all the tasks because of an unwanted task. A larger click area around the robots can be used to solve this problem.

\section{FUTURE WORK}

In the short term we are planning to further analyze the data from the study and to follow up on the various user comments in order to improve our robotic team control mechanisms. We would like to extend the Tractus approach to various practical robotic applications: one approach would be to include tracking mechanisms that will allow us to send a group of real robots through a physical building using the elevator system to move between floors. Such an application can arguably be useful for security or EOD tasks although it is doubtful how well tracking mechanisms or elevator travel would work in realistic disaster or war zones. Another direction is to use the Tractus interface for playing collaborative and realistic games with robots in a physical building environment. Robots can be sent as surrogates for the player, looking for various objects or other players.

In the long term, we are hoping to pursue practical mappings of the Tractus interaction paradigm to monitoring and controlling a group of airborne robots, such as UAVs. We believe that a Tractus, or a Tractus-like interface can be effective in controlling airspace routes and tasks of a group of robots that can flexibly change their 3D position. 


\section{CONCLUSION}

We presented a TUI-based 3D interface enabling a single user to monitor and control a team of independent robots in spatial tasks. By mapping the 3D task to a simple physical interface we implemented an intuitive interaction technique that allows the user to explore 3D space by simply moving the interface up and down, following a drawing board metaphor. We demonstrated the potential of our interface in an EOD-like game using real and simulated robots and designed a study that compared the effectiveness of our 3D Tractus TUI approach to a non-TUI interface. While we believe our implementation and study are valuable, and while qualitative results were positive, we would like to stress that our current quantitative results are inconclusive in suggesting whether our TUI approach is "better" than the nonTUI one, or not.

\section{ACKNOWLEDGEMENTS}

We thank our submission reviewers for their helpful comments and suggestions. We are extremely grateful for the continuous help and support of our colleagues and friends from the uTouch research group, and the Interactions Lab at the University of Calgary. Dr. Tak Shing Fung from the University of Calgary provided us with valuable advice and help regarding the statistical analysis of our study's results. This work was supported by the National Science and Engineering Research Council of Canada (NSERC) Discovery Grant (DG), and Undergraduate Student Research Award (USRA) programs.

\section{REFERENCES}

[1] Chronis, G. and Skubic, M. 2004. Robot navigation using qualitative landmark states from sketched route maps. In Proc. IEEE Intl. Conf. Robotics and Automation, New Orleans, LA, pp. 1530-1535.

[2] Fong, T, Thorpe, C., and Baur, C. 2003. Multi-robot remote driving with collaborative control. IEEE Trans. Industrial Electronics, 50(4):699-704.

[3] Freksa, C., Moratz, R., and Barkowsky, T. 2000. Schematic maps for robot navigation. In C. Freksa, W. Brauer, C. Habel, and K. Wender (eds.), Spatial Cognition II: Integrating Abstract Theories, Empirical Studies, Formal Methods, and Practical Applications, Berlin: Springer, pp. $100-114$.

[4] Guo, C., and Sharlin, E. 2008. Exploring the Use of Tangible User Interfaces for Human-Robot Interaction: A Comparative Study. In Human Factors in Computing Systems, ACM CHI, April 2008, to appear.
[5] Lapides, P., Sharlin, E., Costa Sousa, M., and Streit, L. 2006. The 3D Tractus: A Three-Dimensional Drawing Board. In Proceedings of the First IEEE International Workshop on Horizontal Interactive Human-Computer Systems (TableTop '06), Adelaide, Australia.

[6] Likert, R. 1932. A technique for the measurement of attitudes. In Archives of Psychology, 140:5-53.

[7] Lundberg, C., Barck-Holst, C., Folkeson, J., and Christensen, H.I. 2003. PDA interface for a field robot. In Proc. 2003 IEEE/RSJ Intl. Conf. Intelligent Robots and Systems, Las Vegas, NV, pp. 2882-2887.

[8] Perzanowski, D., Schultz, A.C., Adams, W., Marsh, E., and Bugajska, M. 2001. Building a multimodal human-robot interface. IEEE Intelligent Systems, 16(1):16-21.

[9] Quigley, M., Goodrich, M. A., and Beard, R. W. 2004. SemiAutonomous Human-UAV Interfaces for Fixed-Wing MiniUAVs. Proceedings of IROS 2004, Sendai, Japan, Sept 28Oct 2, 2004.

[10] Setalaphruk, V., Ueno, A., Kume, I., and Kono, Y. 2003. Robot navigation in corridor environments using a sketch floor map. In Proc. IEEE Intl. Symp. Computation Intelligence in Robotics and Automation, Kobe, Japan, pp. $552-557$.

[11] Sharlin, E., Watson, B. A., Kitamura, Y., Kishino, F., and Itoh, Y. 2004. On Tangible User Interfaces, Humans and Spatiality. In Personal and Ubiquitous Computing, special issue on "Tangible Interfaces in Perspective".

[12] Skubic, M., Anderson, D., Blisard, S., Perzanowski, D., and Schultz, A., Using a hand-drawn sketch to control a team of robots, In Auton. Robots, 22 (4), 399-410, 2007

[13] Streit, L., Lapides, P., Costa Sousa, M., and Sharlin, E., 2006. Modeling Plant Variations through 3D Interactive Sketches. In 3rd Eurographics Workshop on Sketch-Based Interfaces and Modeling, SBIM 2006, Vienna, Austria.

[14] Tsang, M., Fitzmaurice, G., Kurtenbach, G., Khan, A., and Buxton, B. 2002. Boom Chameleon: Simultaneous capture of $3 \mathrm{D}$ viewpoint, voice and gesture annotations on a spatiallyaware display. In Proc. UIST 2002, p. 111-120.

[15] Ullmer, B., and Ishii, H. 2000. Emerging Frameworks for Tangible User Interfaces, in IBM Systems Journal, Vol. 9, Nos. 3\&4, 2000, 915-931.

[16] Yanco, H.A., Drury, J. 2004. Classifying human-robot interaction: an updated taxonomy. In 2004 IEEE International Conference on Systems, Man and Cybernetics, Vol. 3, 2841-2846 